cavities of fossil crinoids to be filled with a siliceous substance perfectly injecting their most delicate cellular structure, and which Dr. Hunt, on chemical analysis, found to be a hydrous silicate allied to jollyte. I have since, in examining with the microscope various specimens of limestone in the collection of McGill College, met with a British example of this kind of injection, to which I would wish to direct the attention of your microscopists. It is a specimen of olivaceous, imperfectly crystalline limestone, labelled Llangedoc, Wales. The only distinct fossil which it contains is a small body having the characters of the genus Verticillopora. It is filled, however; with crinoidal fragments and fragments of shells, and, when sliced, displays a few very minute univalves, probably of the genus Murchisonia, and also portions of a sponge-like organism with square meshes. The pores and cavities of many of these fossils are filled with a greenish or brownish finely crystalline silicate, which must have been introduced when the organic bodies were still recent, and which Dr. Hunt has ascertained to have the following composition :-

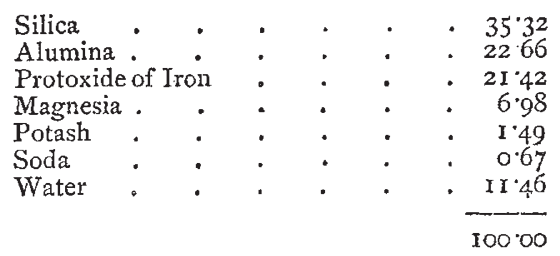

So that this mineral is almost identical with jollyte. The fact that it fills the minute pores and cavities of the fossils can be seen in transparent slices, especially under pola. rised light, and also in decalcified specimens. The filling is not, however, so perfect as in the New Brunswick specimens above alluded to. The best, which I suppose to be Upper Silurian, is worthy of the attention of those who may have access to it, as presenting an interesting example of Silurian fossils preserved in the same way with the Laurentian Eozöon. It affords another palæozoic illustration of a mode of preservation of the structures of fossils, which, though perhaps more prevalent in the Laurentian and Cretaceous than in any intervening periods, is to be met with here and there throughout the geological series, and is of equal interest to the palæontologist and the chemical geologist.

Montreal, June 8

\section{NEW THEORY OF SUN-SPOTS}

A LATE number $(1,835)$ of the Astronomische $N$ achrich ten reproduces from the notices of the Royal Saxon Scientific Society a paper on the above subject by Pro fessor Zöllner. The author believes that he is the first who has attempted to account for the periodicity of the spots by agencies confined to the sun itself, while he rejects the notion of planetary influence to which the phenomenon has been commonly attributed. In this, how. ever, he is not quite correct, for in the April numbers of Cosmos last year there appeared a transcript of a paper read before the Belgian Academy of Sciences, by M. Bernaerts, who tries to explain the various phenomena of the sun.spots without reference to any extra-solar action.

Prof. Zöllner, like M. Bernaerts, accepts the theory of a liquid forming the surface of the sun; but while the Belgian savant considers the spots as perforations in the liquid layer traversed by downpouring currents of gases that had previously risen through the liquid from the gaseous nucleus, Prof. Zöllner believes the spots to be formations of slag or scoriæe caused by a certain local cooling of the liquid surface. Over this glowing liquid is a glowing atmosphere, which contains, in a vaporous state, a portion of the matter belonging to the liquid. The same as on the earth, if this atmosphere is cloudless and calm, radiation and cold are induced; and where this occurs the slag-like products are formed, and spots become visible. But vaporous condensation is also a consequence of the cold. Clouds, therefore, are developed, the radiation is checked, the liquid surface regains its former heat, and the spots are dissolved and disappear; so that the very cause that effects their formation also tends to their dissolution. The repetition of the same operations gives the spots the character of "intermittent phenomena;" but their occurrence, as well as their duration, depends on such a complication of meteorological processes that those phenomena cannot be considered otherwise than as perfectly casual.

The action of a spot on the atmosphere in cooling it, and causing cloudlike condensations that oppose radiation and restore the heat, makes the presence of a great spot unfavourable to the formation of other spots, and Prof. Zöllner arrives at the conclusion that "a sun-spot exerts within a certain area, and according to its size, an influence that prevents or obstructs the formation of other sun-spots." Thus, it appears, he explains the isolation of the spots. But they occur also in groups over a wide extent of surface, and he infers that "the same conditions of the solar atmosphere that induce the formation of a. spot in any one place, prevail in general over a larger space than that occupied by the spot, so that within the area influenced by those favourable conditions, the simultaneous production of other spots is more likely than elsewhere." The size of the spots depends plainly not on the amount of radiation alone, for the slay.like products have cohesive properties like our ice-flates.

I candidly admit that all this is by no means so very plain to me after reading the theory of the isolation of the spots; and I would refer the reader to the original for a better understanding of the two theories relating to the isolation and the grouping, than I have been able to attain to. I would al, 0 refer to the original for the Professor's views of the oscillations of solar temperature and the periodicity of the spots, which he discusses in several paragraphs.

The appearance of the spots in certain zones on both sides of the equator ke explains as the effect of currents in the liquid stratum. He asks us to imagine, in the first place, a motionless, atmosphere-enveloped globe maintained at a constant high temperature; and, after explaining the results, he tells us to fancy such a globe with a liquid envelope heated at bottom by contact with the surface beneath it, and cooled above by radiation. The lower parts of the liquid have a tendency to rise on account of their lower specific gravity, but their ascent anywhere is impossible unless somewhere else a sinking takes place. With equal conditions everywhere prevail:ng, no motion in either direction could occur; but those equal conditions do not exist on the sun, whose axial rotation diminishes the force of gravity at the equator. This therefore favours an ascent of the heated lower portions of the liquid at the equator, and a sinking of the cooler upper parts in the regions of the poles. Two streams are thus induced; one below flowing toward the equator, and one above in a contrary direction. The former as it progresses gains in temperature by contact with the hot surface of the globe; while the latter in its sub-aerial route loses heat by radiation. Thus the polar regions of the sun are made cooler than the equatorial, as has, in fact, been shown by Secchi's investigations.

These movements in the enveloping liquid (fiissigen Umhiullungen) are the cause of atmospheric disturbances, producing in certain places a lowering of temperature and condensation. The fall in temperature is favoured in two ways-by the mixing of the equatorial and polar streams in high latitudes, and by the ascent of an air-current at the equator. As this aircurrent cools in rising its vaporous constituents are partly condensed in the form of clouds. Yet these clouds need not at all be of so low a temperature as to appear to us 
like darkened areas; but, on the contrary, when we consider the high temperature of the sun, we may conceive them to be formed of matter in a glowing state, so that products of condensation such as these could scarcely, if at all, be perceived on the luminous disc of the sun. On the other hand, the author believes that in the cases of the great and still warm planets, Jupiter and Saturn, we see the sun-illumined aqueous clouds that rise in bright belts at the equator. We believe the author's object is to show that, while the visible effects of condensation appear in the atmospheres of Jupiter and Saturn, it is only on the liquid surface that they are exhibited by the sun, and thus its atmosphere remains transparent.

We have, accordingly, in the equatorial zone and in the higher latitudes distinct regions of preponderating atmospheric cloudiness, and between them, like the zones of the trade winds on the earth, lie areas of relative clearness. All this, if I rightly understand the author, is not apparent to the observer, but its effects are seen on the glowing liquid solar surface, where, beneath the unclouded areas, radiation is more induced than in other places, and the formation of sun-spots is the consequence.

To the foregoing causes of atmospheric disturbance must be added the eruptions of hydrogen that are shown by the spectroscope.

On the whole it is the stillness and clearness of certain parts of the atmosphere that induce the formation of spots; and, as the final result of his arguments, Prof. Zöllner sums up as follows :-

"The sun-spots are slag-like products of a cooling process caused by the radiation of heat from the glowing liquid surface, and they dissolve again in consequence of disturbances of equilibrium in the atmosphere which are brought on by themselves. If these disturbances are not merely local, but of more general extent, then, at the times of such general atmospheric disturbances, the formation of new spots is but little favoured, because the essential conditions of a considerable lowering of temperature are wanting, namely-stillness and clearness of the atmosphere. When the atmosphere, after the dissolution of the spots, gradually tranquillises, the process begins anew, and it assumes a periodic character, while the conditions of the solar surface are to be regarded as constant in the mean of lengthened periods. The local distribution of the spots must, according to this theory, depend on the zones of greatest atmospheric clearness, which, as has been shown, are generally coincident with the zones of the greatest development of the spots."

Such, as they appear to me, are Prof. Zöllner's views of the sun's spots; and if, as is quite possible, I have not everywhere succeeded in comprehending him, I freely admit that any misconstruction I have made may be attributable to my own shortcomings rather than to his. At the same time I cannot but regard his style as considerably difficult and diffuse, and not perfect in the logical concentration which is so necessary for the clear enunciation of a theory. In some points his conclusions scem, undoubtedly, to agree with observations-for instance, as regards the vaporous masses that are formed over the spots, and which appear sufficiently attested by their strong absorption lines in the spectrum. In the main, however, I cannot say, so far as I may venture to give an opinion, that he has been more successful than other theorists on the same subject; and among several objections which have occurred to me as affecting his views, I will venture to state the following :-

I. Regarding the establishment of currents in the liquid envelope, Prof. Zöllner affirms that in this way alone could the more heated and specifically lighter portions of the liquid at the bottom make their way to the surface; but this appears to me incorrect when. we consider that in freezing water there is an interchange of the upper and lower strata until congelation begins, and this without the intervention of currents.
2. He defines the spots as scoriaceous products floating on the liquid surface. 'The liquid, however, is moving in a current from the equator poleward, and, if so, I would ask how is it that the spots show no tendency to be carried along with it in that direction? I do not believe that any such general tendency has been observed.

3. He makes no attempt to account for the very striking and suggestive appearances of the penumbra, which led Wilson to regard the spots as openings or depressions in the photosphere. Neither does he try to explain the distinct boundaries of the nucleus, the umbra, the penumbra, the light bridges, nor the deeper shading of the penumbra round its exterior limits.

4. The current cools in its advance poleward, and the polar regions are, as the professor tells us, the coolest parts of the sun. Then if, according to his theory, the spots are the products of cold, why do they not increase in development up to the poles? He assumes, indeed, that the cold induced in the polar regions produces clouds in the atmosphere, which are unfavourable to the production of spots ; but they are so only as they check radiation and contribute to heat, and if, notwithstanding this, the polar regions are still found to be the coldest, and if cold is the cause of the spots, there seems a defect in the hypothesis. The professor points to an analogy between the spot zones and the clear zones of the trade winds of the earth. The cases are, however, very different ; for our own atmosphere is subject to external influences, namely, the action of the sun, as well as those belonging to the earth itself.

Of course, anything from the eminent pen of Prof. Zöllner must be received with the utmost respect, but I conceive that his theory of the sun-spots, as I have attempted partly to show, presents many difficulties; and I cannot avoid stating my humble belief that, notwithstanding all that has been thought and written on the subject, and in spite of the modern discoveries of some of the constituent elements of the sun, we are but little nearer a true conception of its organisation or economy than the theorists of the days of Hipparchus.

\section{J. BIRMINGHAM}

\section{PROFESSOR TYNDALL ON THEORIES OF DISEASES}

WE reprint this article from the British Medival foumal, since it shows how closely connected are the most abstract inquiries with the most practical questions :-

"The surest basis for Medicine is upon the broad foundations of exact scientific observation; and we shall all welcome such contributions as so able a physicist as Professor Tyndall can make either to our knowledge or to our facilities for testing the foundations of our beliefs. The electric beam, which has in his hands played a large part in many able investigations and demonstrative experiments, was lately brought into play to demonstrate the ubiquity of dust in the atmosphere. To some very charming experiments $\mathrm{Mr}$. Tyndall joined some theories which, if capable of proof, were yet not demonstrated by anything which he said or did. The ubiquity of these airborne particles was perfectly well known, and their illumination by the electric beam, while it has given a more complete demonstration of their presence than was otherwise obtainable, has not added anything to our knowledge of their chemical or biological relations. His experiments, however, have had the valuable effect of demonstrating the uses of cotton-wool as a filter for them, and the advantage of inspiring him with interest in a subject in which we can but be pleased that one of the most brilliant of investigators and expositors of physical science should be interested - the investigation of the origin of zymotic disease. 